\title{
Differential constraints for the Kaup - Broer system as a reduction of the 1D Toda lattice
}

\author{
A K Svinin $\dagger$ \\ Institute of System Dynamics and Control Theory, Siberian Branch of Russian \\ Academy of Sciences, P.O. Box 1233, 664033 Irkutsk, Russia
}

\begin{abstract}
It is shown that some special reduction of infinite 1-D Toda lattice gives differential constraints compatible with the Kaup - Broer system. A family of the travelling wave solutions of the Kaup - Broer system and its higher version is constructed.
\end{abstract}

Short title: Differential constraints for the Kaup - Broer system as a reduction of the 1D Toda lattice

October 24, 2018

$\dagger$ E-mail address : svinin@icc.ru 


\section{Introduction}

The goal of this paper is to show that differential constraints compatible with the Kaup

- Broer system [1], 2]

$$
\begin{aligned}
& \frac{\partial S}{\partial t_{2}}=-S_{x x}+2 S S_{x}+2 R_{x} \\
& \frac{\partial R}{\partial t_{2}}=R_{x x}+2(R S)_{x}
\end{aligned}
$$

can be derived as a special reduction of the 1D Toda lattice and to select some class of travelling wave solutions of it and its higher counterpart.

It is well known that the Kaup - Broer system is applied in hydrodynamics. It appears as a model equation for nonlinear water waves. In turn, the Kaup - Broer hierarchy appears to be related with one-Hermitian matrix model and can be extracted from Toda lattice hierarchy where the first flow parameter is treated as the space coordinate [3].

As is known, the hierarchy of the Kaup - Broer system is intimately related to the Kadomtsev - Petviashvili hierarchy and can be interpreted as a special reduction of it whose evolution equations are coded by the Lax equation

$$
\frac{\partial \mathcal{L}}{\partial t_{p}}=\left[\mathcal{L}_{+}^{p}, \mathcal{L}\right]
$$

on the monic pseudodifferential operator $\mathcal{L}=\partial+\sum_{i=1}^{\infty} U_{i}(x) \partial^{-i}$. The corresponding Lax operator is constrained by the condition

$$
\mathcal{L}=\partial+\sum_{i=1}^{\infty} U_{i}[S, R] \partial^{-i}=\partial+R(\partial-S)^{-1}, \quad \partial=\frac{\partial}{\partial x} .
$$

The relationship with the Toda lattice hierarchy provides evolution equations of the Kaup - Broer hierarchy by the property of the existence of discrete symmetries generated by similarity transformation

$$
\overline{\mathcal{L}}=\left(\partial-S^{0}\right) \mathcal{L}\left(\partial-S^{0}\right)^{-1}
$$

where $S^{0}=S+\partial \ln R$. More explicitly, we have

$$
\bar{S}=S+\frac{R_{x}}{R}, \bar{R}=R+S_{x}+\frac{R_{x x}}{R}-\frac{R_{x}^{2}}{R^{2}} .
$$

One of the effective methods of searching for exact solutions is to select them by means of differential constraints [4], [5]. In this article we find the differential constraints for the Kaup - Broer system and its higher counterpart. To this aim, we use a reduction of the Toda lattice which is among many found recently in [6]. These constraints select a broad class of traveling wave solutions. 
This paper is organized as follows. In section 2, we recall definition of the 1D Toda lattice and its relationship with the Kaup - Broer hierarchy. In section 3, we exhibit a denumerable class of reductions of the infinite 1D Toda lattice [6]. Next, we discuss the case $n=1$. We show that in this case the reduced system serves as differential constraints for the Kaup - Broer system and its higher counterpart. Also, we show that these differential constraints isolate the solutions of travelling wave type.

\section{Preliminaries}

We give here background information on the 1D Toda lattice and its relationship with the Kaup - Broer hierarchy. Also we recall the notion of differential constraints compatible with a given evolutionary system of partial differential equations.

The Toda lattice, as is known, can be represented as the consistency condition of linear auxiliary equations

$$
\begin{aligned}
& L\left(\psi_{i}\right)=\psi_{i+1}+a_{0}(i) \psi_{i}+a_{1}(i) \psi_{i-1}=z \psi_{i}, \\
& \psi_{i x}=A\left(\psi_{i}\right)=\psi_{i+1}+a_{0}(i) \psi_{i}, \quad i \in \mathbf{Z} .
\end{aligned}
$$

The corresponding Lax equation $\partial(L)=[A, L]$ is equivalent to equations of the onedimensional Toda lattice

$$
\begin{aligned}
& a_{0 x}(i)=a_{1}(i+1)-a_{1}(i), \\
& a_{1 x}(i)=a_{1}(i)\left(a_{0}(i)-a_{0}(i-1)\right) .
\end{aligned}
$$

Introducing variables $u_{i}$ by

$$
a_{0}(i)=-u_{i x}, \quad a_{1}(i)=e^{u_{i-1}-u_{i}}
$$

one can rewrite the Toda lattice (6) in usual form:

$$
u_{i x x}=e^{u_{i-1}-u_{i}}-e^{u_{i}-u_{i+1}} .
$$

From (5) one can easy derive that each wavefunction $\psi_{i}$ satisfies the linear equation

$$
\mathcal{L}_{i} \psi_{i}=z \psi_{i}
$$

where

$$
\mathcal{L}_{i}=\partial+a_{1}(i)\left(\partial-a_{0}(i-1)\right)^{-1}
$$

It is easy to show that, by virtue of (6) the operators $\mathcal{L}_{i}$ are related by the invertible gauge transformation

$$
\mathcal{L}_{i+1}=\left(\partial-a_{0}(i)\right) \mathcal{L}_{i}\left(\partial-a_{0}(i)\right)^{-1} .
$$

Fixing any value $i=i_{0} \in \mathbf{Z}$ one identifies

$$
a_{1}\left(i_{0}+1\right)=R, \quad a_{0}\left(i_{0}\right)=S .
$$


From The equations of Toda lattice (6) one can easily extract the symmetry transformation (4) generated by the shift $i \rightarrow i+1$.

Let us also briefly discuss the notion of differential constraints compatible with a given system of partial differential equations. Let $E$ be a partial differential system with respect to functions $u^{1}, \ldots, u^{m}$ of two variables, say $t \in \mathbf{R}^{1}$ and $x \in \mathbf{R}^{1}$. The notation $[E]$ stands for union of $E$ and its differential consequences with respect to $x$. In what follows we restrict ourselves by consideraton only evolutonary equations

$$
u_{t}^{i}=F^{i}\left[u^{1}, \ldots, u^{m}\right]
$$

where $F^{i}$ are some (analytic) differential functions of $u^{1}, \ldots, u^{m}$. Let the system (11) be supplemented by differential constraints $H$

$$
h_{j}\left[u^{1}, \ldots, u^{m}\right]=0, \quad j=1, \ldots, p, \quad p \leq m .
$$

One says that the differential constraints (12) define an invariant manifold of the system (11) if

$$
\left.D_{t}\left(h_{j}\right)\right|_{[E] \cap[H]}=0, \quad j=1, \ldots, p,
$$

where $D_{t}$ denotes total derivative with respect to $t$. Equations (13), whose solutions are some collections of differential functions $\left\{h_{1}, \ldots, h_{p}\right\}$, are reffered to as determining ones.

It is quite difficult to use determining equations (13) with $h_{j}$ in general form. However, the situation is considerably simplified if (12) can be resolved with respect to higher-order derivatives as

$$
\left(u^{j}\right)_{x}^{\left(N_{j}\right)}=S^{j}\left(t, x, u^{1}, \ldots, u^{m}, u_{x}^{1}, u_{x}^{2}, \ldots\right) .
$$

In this case a simple practical recipe to solve the determining equations (13) consists of successively replacing $\left(u^{j}\right)_{x}^{\left(N_{j}\right)} \rightarrow S^{j}$.

It is common of knowledge that the problem of finding all differential constraints compatible with a given equation (system of equations) can be more complicated than solving these equations. In practice, it is better to restrict oneself to finding differential constraints in some fixed classes. Regarding to the development of regular methods for constructing differential constraints, see [5], [10].

\section{Reductons of the 1D Toda lattice, differential constraints and travelling wave solutions of the Kaup - Broer system}

Recently, we have proposed an infinite class of reduction of the Toda lattice [6]. They are specified by constraints

$$
\begin{aligned}
& -a_{0}(i)-\ldots-a_{0}(i+n-1)=a_{1}(i) a_{1}(i+1) \ldots a_{1}(i+n), \\
& i \in \mathbf{Z}, \quad n \in \mathbf{N}
\end{aligned}
$$


or

$$
u_{i x}+\ldots+u_{i+n-1, x}=e^{u_{i-1}-u_{i+n}} .
$$

For any fixed $i=i_{0} \in \mathbf{Z}$, introduce a finite number of functions $\left\{q_{1}, \ldots, q_{n+1}\right\}$ identifying

$$
q_{1}=u_{i_{0}}, q_{2}=u_{i_{0}+1}, \ldots, q_{n+1}=u_{i_{0}+n} .
$$

From (8) and (15) we derive the finite-dimensional system

$$
\begin{aligned}
& q_{1 x x}=\left(q_{1 x}+\ldots+q_{n x}\right) e^{q_{n+1}-q_{1}}-e^{q_{1}-q_{2}}, \\
& q_{k x x}=e^{q_{k-1}-q_{k}}-e^{q_{k}-q_{k+1}}, \quad k=2, \ldots, n, \\
& q_{n+1, x x}=e^{q_{n}-q_{n+1}}-\left(q_{2 x}+\ldots+q_{n+1, x}\right) e^{q_{n+1}-q_{1}}
\end{aligned}
$$

togegher with discrete symmetry transformation generated by the shift $i \rightarrow i+1$

$$
\bar{q}_{1}=q_{2}, \ldots, \bar{q}_{n}=q_{n+1}, \bar{q}_{n+1}=q_{1}-\ln \left[q_{2 x}+\ldots+q_{n+1, x}\right] .
$$

In what follows, we restrict our attention to the case $n=1$. We have

$$
q_{1 x x}=q_{1 x} e^{q_{2}-q_{1}}-e^{q_{1}-q_{2}}, \quad q_{2 x x}=e^{q_{1}-q_{2}}-q_{2 x} e^{q_{2}-q_{1}} .
$$

Observe that the system (19) can be cast into canonical Hamiltonian setting. Generalized momenta are introduced as

$$
p_{1}=-q_{2}^{\prime}-\frac{1}{2} e^{q_{2}-q_{1}}, \quad p_{2}=-q_{1}^{\prime}-\frac{1}{2} e^{q_{2}-q_{1}} .
$$

One can verify that equations (19) are equivalent to Hamiltonian system

$$
q_{i x}=\frac{\partial H}{\partial p_{i}}, \quad p_{i x}=-\frac{\partial H}{\partial q_{i}}, \quad i=1,2,
$$

where

$$
H=-\left(p_{1}+\frac{1}{2} e^{q_{2}-q_{1}}\right)\left(p_{2}+\frac{1}{2} e^{q_{2}-q_{1}}\right)+e^{q_{1}-q_{2}} .
$$

The first two integrals of the (19) are $H$ and $P=p_{1}+p_{2}$. It is simple exersice to check that $H$ and $P$ are in involution with respect to standard Poisson bracket. So we can conclude that the equations (19) establish Hamiltonian system integrable in the sense of Liouville theorem [7]. It is natural to suppose that all systems (17) are Liouville-integrable.

To proceed, we need to express variables $R$ and $S$ via $q_{1}$ and $q_{2}$. Taking into account (17), (10) and (16) one obtains

$$
\begin{aligned}
& S=a_{0}\left(i_{0}\right)=-u_{i_{0}, x}=-q_{1 x}, \\
& R=a_{1}\left(i_{0}+1\right)=e^{u_{i_{0}}-u_{i_{0}+1}}=e^{q_{1}-q_{2}} .
\end{aligned}
$$


From Toda lattice equations (6), by virtue of (14), we obtain the following differential equations:

$$
\begin{aligned}
& S_{x}=a_{0 x}\left(i_{0}\right)=a_{1}\left(i_{0}+1\right)-a_{1}\left(i_{0}\right)=a_{1}\left(i_{0}+1\right)+\frac{a_{0}\left(i_{0}\right)}{a_{1}\left(i_{0}+1\right)}=R+\frac{S}{R}, \\
& \bar{S}_{x}=a_{0 x}\left(i_{0}+1\right)=a_{1}\left(i_{0}+2\right)-a_{1}\left(i_{0}+1\right) \\
& =-\frac{a_{0}\left(i_{0}+1\right)}{a_{1}\left(i_{0}+1\right)}-a_{1}\left(i_{0}+1\right)=-\frac{\bar{S}}{R}-R
\end{aligned}
$$

where $\bar{S}$ is given by (雨). The latter, as can be checked, in more explicit form reads as a pair of ordinary differential equations

$$
\begin{aligned}
& S_{x}=R+\frac{S}{R}, \\
& R_{x x}=\frac{R_{x}^{2}}{R}-\frac{R_{x}}{R}-2 R^{2}-2 S .
\end{aligned}
$$

One can verify that differential substitution (21) indeed maps solutions of the system (19) into solutions of (22).

By using determining equations (13) one can verify that equations (22) serve as differential constraints compatible with the Kaup - Broer system (1) and its higher version

$$
\begin{aligned}
& \frac{\partial S}{\partial t_{3}}=S_{x x x}-3 S S_{x x}-3 S_{x}^{2}+6(S R)_{x}+3 S^{2} S_{x} \\
& \frac{\partial R}{\partial t_{3}}=R_{x x x}+6 R R_{x}+3 S R_{x x}+3 S_{x} R_{x}+3\left(S^{2} R\right)_{x} .
\end{aligned}
$$

We conjecture that relations (22) play the role of differential constraint for all members of the Kaup - Broer hierarchy. Following question arises: solutions of what kind are isolated by differential constraints (22)? The following proposition is helpful to answer this question.

Proposition. By virtue of differential constraints (22) following relations hold:

$$
\begin{aligned}
& \frac{\partial S}{\partial t_{2}}=P S_{x}, \quad \frac{\partial R}{\partial t_{2}}=P R_{x} \\
& \frac{\partial S}{\partial t_{3}}=\left(E+P^{2}\right) S_{x}, \quad \frac{\partial R}{\partial t_{3}}=\left(E+P^{2}\right) R_{x},
\end{aligned}
$$

where

$$
P=2 S+\frac{R_{x}}{R}-\frac{1}{R}, E=-S^{2}+R-S \frac{R_{x}}{R}
$$

are two first integrals of the system (22).

This proposition is proved by straightforward calculation. 
Remark 1. The integrals $P$ and $E$ are $P=p_{1}+p_{2}$ and $H$ (20) expressed in terms of variables $S$ and $R$.

Taking into account the proposition above, it is natural to suppose that there exists an infinite collection of polynomials $K_{l}(E, P)$ such that by virtue (22), relations of the kind as in (24) and (25) are valid, i.e.

$$
\frac{\partial S}{\partial t_{l}}=K_{l}(E, P) S_{x}, \quad \frac{\partial R}{\partial t_{l}}=K_{l}(E, P) R_{x} .
$$

Thus $K_{1}=1, K_{2}=P$ and $K_{3}=E+P^{2}$.

Next, we observe that, by virtue of (22), $E$ and $P$ also do not depend on $t_{l}$. Take for example $E$. Taking into account (26) we have

$$
D_{t_{l}}(E)=\frac{\partial E}{\partial R} R_{x} K_{l}+\frac{\partial E}{\partial R_{x}} R_{x x} K_{l}+\frac{\partial E}{\partial S} S_{x} K_{l}=D_{x}(E) K_{l}=0,
$$

where $D_{t_{l}}$ and $D_{x}$ stands for total derivative with respect to corresponding argument. The proposition above and this observation prove that differential constraints (22) select simultaneous solution of the systems (11) and (23) in the form of travelling wave

$$
S=S(\xi), \quad R=R(\xi),
$$

where $\xi=x+P t_{2}+\left(E+P^{2}\right) t_{3}+\xi_{0}$, where $\xi_{0}$ is some constant (it may depend on $t_{4}$, $\left.t_{5}, \ldots\right)$.

Thus, to find a profile of travelling wave (27) which is simultaneous solution of (1) and (23) we need to solve ordinary differential equations

$$
\begin{aligned}
& S^{\prime}=R+\frac{S}{R}, \\
& R^{\prime \prime}=\frac{R^{\prime 2}}{R}-\frac{R^{\prime}}{R}-2 R^{2}-2 S .
\end{aligned}
$$

with some initial conditions $\left(R_{0}=R(0), R_{1}=R^{\prime}(0), S_{0}=S(0)\right.$ ).

Remark 2. In (27) $P$ and $E$ are understood as some values $P=P_{0}$ and $E=E_{0}$ of first integrals corresponding to a particular solution of (28).

To conclude this section, let us discuss Painlevé property for system (28). Simple analysis shows that it passes Painlevé test. In addition, the system (28) has a formal "general" solution in the form of pole-like expansion

$$
\begin{aligned}
& S(\xi)=\frac{1}{\xi}\left(1+c_{1} \xi+c_{2} \xi^{2}-\frac{1}{2} \xi^{3}-\left(\frac{2}{5} c_{1}+\frac{1}{5} c_{2}^{2}\right) \xi^{4}+O\left(\xi^{5}\right)\right) \\
& R(\xi)=\frac{1}{\xi^{2}}\left(-1+c_{2} \xi^{2}-\left(\frac{1}{5} c_{1}+\frac{3}{5} c_{2}^{2}\right) \xi^{4}+O\left(\xi^{5}\right)\right)
\end{aligned}
$$

with two arbitrary constants $c_{1}$ and $c_{2}$. Fuchs indices are $-1,1$ and 2 . 
Suppose now that solution (27) does not depend on $t_{2}$. It is equivalent to assuming that $P=0$. In turn this requires that

$$
S=-\frac{R^{\prime}}{2 R}+\frac{1}{2 R}
$$

It is easy to check that relation (29) properly defines reduction of system (28) to the equation

$$
R^{\prime \prime}=\frac{R^{\prime 2}}{R}-\frac{1}{R}-2 R^{2}
$$

which is particular case of Panlevé 12 equation 8]

$$
R^{\prime \prime}=\frac{R^{\prime 2}}{R}+\frac{\alpha}{R}+\beta+\gamma R^{2}+\delta R^{3}
$$

with $\alpha=-1, \beta=0, \gamma=-2$ and $\delta=0$.

\section{Discussion}

In this paper we have derived differential constraints for the Kaup - Broer system via reducton of the infinite $1 \mathrm{D}$ Toda lattice. It is shown that these differential constraints select a family of travelling wave solutions.

Ablowitz - Ramani - Segur (ARS) conjecture states that any theoretical group reduction of an integrable system of partial differential equations will have the (generalized) Painlevé property [9]. We believe that ARS conjecture could be extended on differential constraints. The example of the system (28) exhibited in this paper supports this conjecture.

\section{Acknowledgments}

We are grateful to the referees for carefully reading the manuscript and for their remarks which enabled us to improve the presentation of the paper.

The author wishes to thank the Editorial Board for the invitation to contribute the paper to this issue. This research has been partially supported by INTAS grant 2000-15.

\section{References}

[1] Kaup D J 1975 Progr. Theor. Phys. 54396

[2] Broer L J F 1975 Appl. Sci. Res. 31377

[3] Bonora L and Xiong C S 1992 Phys. Lett. B 285191 Bonora L and Xiong C S 1993 Int. J. Math. Phys. 82973

[4] Sidorov A F, Shapeev V P and Yanenko N N 1984 Method of differential constraints and its applications in gas dynamics (Novosibirsk: Nauka) (in Russian) 
[5] Andreev V K, Kaptsov O V, Pukhnachev V V and Rodionov A A 1994 Application of grouptheoretic methods in fluid dynamics (Novosibirsk: Nauka) (in Russian)

[6] Svinin A K 2000 Teoreticheskaya i Matematicheskaya Fizika 124419 (in Russian)

[7] Liouville J 1855 J. Math. Pures Appl. 20137

[8] Ince E L 1956 Ordinary differential equations (New York: Dover)

[9] Ablowitz M J, Ramani A and Segur H J. Math. Phys. 21 715, 1006

[10] Kaptsov O V 1998 Mat. Sbornik 189103 (in Russian) 